\title{
Research on Precision Marketing Strategy in Big Data Era
}

\author{
Juxiang Zhang \\ Tan Kah Kee College, Xiamen University, ZhangZhou, China \\ 14827686@qq.com
}

\begin{abstract}
Keywords: Big data, Data analysis, Model building, Precision marketing.
Abstract. With the development of e-commerce and the rise of big database analysis, enterprises pay more and more attention to big data marketing. This paper discusses the basic process and content of big data marketing from several aspects, such as data collection, data analysis and model construction, precision marketing application based on big data. Some precision marketing strategies concerning customer segmentation, personalized product recommendation, precision marketing information dissemination, market forecast and brand communication are put forward.
\end{abstract}

\section{Introduction}

With the development of new technologies, such as mobile Internet, the amount of data in the world is increasing explosively, affecting the competitive form in different trades. Domestic and foreign Internet giants began to widely use big data in the daily marketing management. For example, Alibaba, Baidu, Amazon and other companies analyze customers' daily network behaviors; dig the potential needs of customers in order to establish a new marketing model with the characteristics of precision marketing.

Currently, China's e-commerce is in the stage of rapid development. In 2015, online retail sales are 3.8 trillion $\mathrm{RMB}$, increasing $36.2 \%$ over last year, occupied $12.6 \%$ of the total retail sales of consumer goods ${ }^{[1]}$. When more and more traditional enterprises apply e-commerce, they recognize the value of big data analysis in the network marketing. Thus, Discussion on how to implement the precision marketing based on big data will help to improve sales performance for enterprises.

\section{Relevant concepts}

Big data is a term for data sets that are so large or complex that traditional data processing application software is inadequate to deal with them. Challenges include capture, storage, analysis, data curation, search, sharing, transfer, visualization, querying, and updating and information privacy ${ }^{[2]}$.

Usually characteristics of big data are described as 4"V": volume, variety, velocity, and value ${ }^{[3]}$. Volume means the huge amount of data which from the TB level jumped to the PB level. Variety refers to a wide variety of data sets such as web logs, videos, pictures, location information, etc. Velocity means the high processing speed that can quickly obtain valuable information from various types of data. Value refers to accurate data analysis will bring a high value return.

\section{Data collection}

In the process of big data marketing, enterprises first need to obtain sufficient data, and then build a characteristic data model based on data analysis, and finally utilize model for precision marketing ${ }^{[4]}$. Detailed process is shown in Fig.1. 


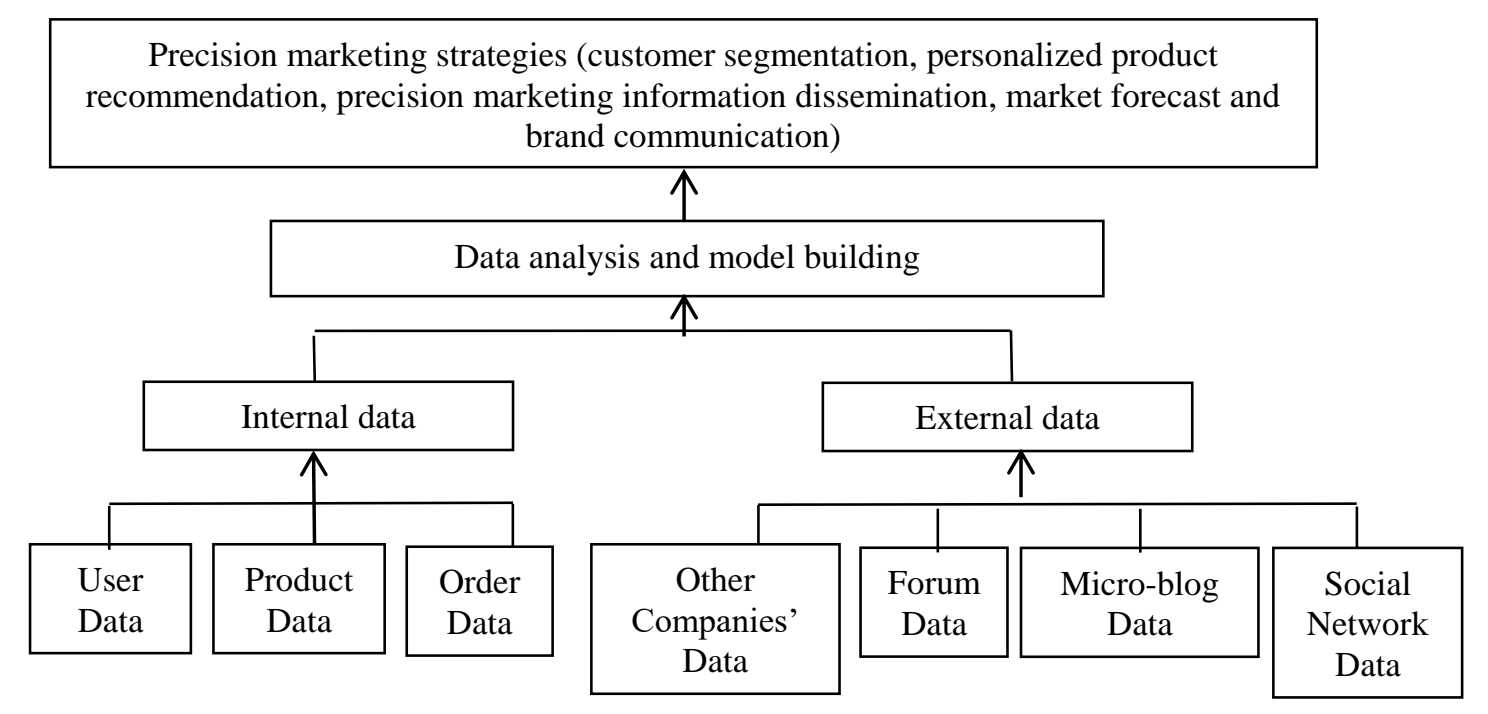

Fig.1. The basic process of big data marketing

While mining data, enterprises not only need to analyze internal data about products, customers and order data, but also need pay attention to external data collection and analysis. The establishment of large database has the following three methods.

\subsection{Self-built model}

According to their own needs, Enterprises build a consumer-centric and market-oriented database by themselves. Usually, data can be collected through the following two ways. First of all, companies sort out internal data of business operation, including product data, sales data, distribution channels data, customer basic information data and etc. Secondly, companies acquire multi-dimensional user behavior data via internet. The main method is web crawler technology. Through this technology, network user behavior records can be gained, including their behaviors in various forums, social networks, micro blogging and etc. Self-built database lays a foundation for subsequent precision marketing.

\subsection{Resource-sharing model}

Through sharing data resources between enterprises, a more comprehensive database can be built. For example, as an e-commerce platform, Alibaba doesn't have social platform. Therefore, except consumption habits it is difficult for Alibaba to effectively grasp user interests and other habits. With the cooperation of Sina micro-blog, Alibaba is able to get user's social information for further precision product recommendation. Similarly, Sina micro-blog understands the user's consumptive habits through Alibaba e-business platform, so that it can push more accurate internet advertising.

\subsection{Third-party data supply model}

The third party data service provider refers to companies that collect data from all walks of life and provide data processing and solutions. They integrate a large amount of data by collecting national statistical yearbook, business Yearbook and analysis report, consumer behavior survey and other professional reports. Hence, enterprises with no database are able to obtain data through the third party data service provider in a paid way ${ }^{[5]}$.

In addition, some internet companies provide big data analysis tools for small companies and individuals. For example, Baidu launched Baidu search data index based on mass search data; Taobao launched Taobao index based on users' search and shopping data. Making full use of these indexes can help companies better understand industry demand and status of industry sales. No matter what kind of data acquisition method, data resource integration has become a trend. Only by integrating 
data resources, a real consumer-oriented large database can be built, which is the premise of precision marketing.

\section{Data analysis and model building}

Precision marketing requires insight into the needs of consumers through analyzing consumer data. In general, user data analysis mainly includes three parts: basic information analysis, access behavior analysis and social relationship analysis.

\subsection{User basic information analysis}

E-business users are usually registered users. When users finish the website registration, enterprises will have users' basic information, including name, gender, age, telephone number and etc. Once users begin shopping, home addresses and other information can be gotten.

After connecting users' basic information and orders and doing correlation analysis and cluster analysis, main consumer groups can be described. Possible association between product color preferences, performance preferences and user gender, age and region can be found as well. Accordingly, the corresponding relational model can be built.

\subsection{User access behavior analysis}

The analysis of user access behavior is mainly to analyze the relationship between access behavior and behavioral effect. User access behaviors mainly include content of access pages, page retention time, keyboard input, mouse click, touch and click on the mobile terminal and etc. The behavioral effect mainly includes click rate of online shop, click rate of the product, conversion rate about click to buy, buy back rate, page click ranking, and etc. The analysis of these behavioral data can provide valuable advice for online shop optimization design and sales promotion.

\subsection{User social relationship analysis}

Social network users usually have similar interests and often recommend products for each other. Thus, taking advantage of users' social relationships could effectively target potential customers with lower cost. Viral marketing is a typical way to use social relationships for marketing.

Usually Social relationships are divided into strong and weak relationships. QQ group and WeChat circle belong to strong relationships. Users in strong relationships network are usually familiar with each other. Although the number of users in strong relationships network is generally limited, the possibility of becoming a target customer is great because of its strong social network homogeneity. Micro-blog is usually a weak relationship with a strong social heterogeneity between each other; therefore, the probability of becoming a target customer is relatively low. However, usually there are a great number of users in a weak relationship network including friends of friends, or even strangers. Thus information can be quickly spread in such a social communication network. Through big data analysis, users' strong relationship and weak relationship maps can be drawn for companies.

Through the user basic information analysis, access behavior analysis and social relationship analysis, a user portrait model can be established ${ }^{[6]}$. This model relates a series of online consumer behaviors, including browsing, click, reviews, comments, videos and pictures, so as to restore the consumer and draw accurate portrait of the consumer. Through the user portrait model, enterprises can better understand the consumer's personality, habits, attitudes and other information; dig into the needs of consumers for making marketing decisions.

\section{Precision marketing application based on big data}

Precision marketing can be applied based on big data analysis and model building, including personalized product recommendation, advertising optimization, customer segmentation, market forecasts, brand communication and etc. 


\subsection{Customer segmentation}

The traditional customer segmentation is mainly based on customers' gender, age, geographical and other basic division characteristics. Since the division is rough, there is often a big difference in the customer group. However, based on big data analysis customers with similar buying behavior can be divided into a subdivision of the customer groups ${ }^{[7]}$. Enterprises are able to extract characteristics of their purchasing preferences, and accordingly carry on targeted product development and promotion.

\subsection{Personalized product recommendation}

Recommendation system has become a key application of big data marketing. Enterprises can build product association models based on user transactions to recommend products. Since the product association model needs a large number of online transaction data, it is more suitable for hot-sale products to further expand the target customers. For the new product, enterprises can depend on the user portrait model to select target customers, which is based on user characteristics, shopping preferences and product characteristics. Once target customers are chosen, information can be push forward to them through the online page, e-mail, WeChat corporate public numbers and other means.

\subsection{Precision marketing information dissemination}

Precision marketing information dissemination refers to push business information concerning product advertising, promotional activities and other messages to target audiences, so as to attract their attention, click and reading. Precision marketing information dissemination needs to consider who the target audiences are and what contents need to be pushed.

\subsubsection{Real time communication based on RTB}

RTB is the acronym of "Real Time Bidding". It is an accurate means of communication supported by big data technology. The principle of RTB is to use cookie to record all the user behaviors on the web, such as online search, browse products, click on the advertising window or link and so on, and analyze the user's preferences. When the user visits the website next time, the system will push advertising in line with his interests. Hence, RTB not only save the cost of advertising for companies, but also make advertising more accurate and save time for customers, which achieving win-win between enterprises and customers.

For example, through big data technology, a company selling maternal and child supplies finds that a user regularly buys Infant Formula online. Based on the user's purchase frequency, product information and other valid data, the company can judge that a child was just born in the family. Therefore, the company will push other products ads about early literacy, baby toys, and baby clothes and so on when the user shops again. These ads would not cause the user's resentment; on the contrary, the user would be interested in these ads, click on and browse ads. Consequently, precise advertising based on RTB helps to achieve efficient marketing promotion with low cost.

\subsubsection{Content dissemination based on social interaction}

With the rapid development of social networks, people spend a lot of time chatting, making friends, telling the mood, share shopping experience on the social platform, and these social behaviors will have a certain impact on other circle members. So, enterprises can take advantage of the autonomy of social users and trust in social circles to create content dissemination. Enterprises can take advantage of big data technology to find opinion leaders of social circles to expand the scope of influence, such as micro-blog star's fan effect.

Since circle members are more inclined to trust each other owing to some common characteristics, it is a good opportunity for enterprises to spread some personalized product information and marketing content in social circles.

\subsection{Market prediction}

Market forecast is a long-term concern in the field of data analysis and data mining. The traditional market forecast usually adopts the method of sampling, statistics and so on. Because of the limited amount of data, the forecasting accuracy is not high. Through big data analysis, industry trends and 
market demand trends can be more quickly and comprehensively predicted, especially the product demand forecast of market segments.

\subsection{Brand communication}

The brand has intangible and irreplaceable value, and enterprises can benefit from it in the market competition. Interpersonal communication is an efficient way to enhance brand reputation. Enterprises can take advantage of user social relationship maps to identify the key users, who are active in various social circles, so that the speed of information dissemination may grow exponentially. On the other hand, enterprises can identify the direction of transmission through the effectiveness analysis of brand communication based on big data, such as the spread trend analysis, content characteristics analysis, network public opinion analysis and so on.

\section{Conclusion}

With the development of information technology, the competition among enterprises will be gradually transformed into the competition of data acquisition and analysis in the future. Enterprises need to timely and accurately understand consumer behaviors, and grasp consumption trend in order to win consumers. Therefore, data collection, data analysis and prediction are gradually playing an important role in business operation. Precision marketing strategies based on big database analysis, which include customer segmentation, personalized product recommendation, precision marketing information dissemination, market forecast and brand communication, will contribute to enhance enterprises competitiveness.

\section{References}

[1] China Electronic Commerce Research Center, China online shopping industry monitoring report in 2016, http://www.100ec.cn/detail--6342861.html

[2] Wikipedia.https://en.wikipedia.org/wiki/Big_data

[3] Duan Xiaochen, A study on the marketing strategy of China e-commerce in big data era, Journal of Xi'an Petroleum University, vol.4, pp. 48-53, 2016.

[4] Peng Haijing, Research on the strategy of big data marketing of minor enterprises, Commercial Economy, vol.7, pp. 15-17, 2016.

[5] Xue Zhenggui, Research on marketing system reconfiguration based on big data, Journal of Heihe University, vol. 5, pp. 64-67, 2015.

[6] Zhang Peng and Liu Yijing, Portrait of the consumer, Sales and Marketing, vol.9, pp. 30-32, 2013.

[7] Li Xiaolong and Feng Junwen, Research on precision marketing strategy for e-business in the environment of big data, Value Engineering, vol.3, pp. 31-33, 2016. 